\title{
Validation of Y-ancestor time calculators for forensic familial searching
}

\author{
Sofie Claerhout ${ }^{a, *}$, Charlotte Defraye ${ }^{a}$, Ronny Decorte ${ }^{a, b}$ \\ ${ }^{a}$ KU Leuven, Forensic Biomedical Sciences, Department of Imaging \& Pathology, Leuven, Belgium \\ b UZ Leuven, Laboratory of Forensic genetics and Molecular Archaeology, Leuven, Belgium \\ ${ }^{*}$ Corresponding author at the Laboratory of Forensic Genetics and Molecular Archaeology, \\ Kapucijnenvoer 33, Leuven, Belgium. E-mail: sofie.claerhout@kuleuven.be
}

\begin{abstract}
When no suspects are identified in a forensic case as the donor of a biological trace, familial searching through the $\mathrm{Y}$-chromosome can be used as an identification method to find paternally related males of the perpetrator. When a close $\mathrm{Y}$-haplotype match is identified, the time to their most recent common ancestor (tMRCA) can be estimated to reconstruct their genealogy. To date, three online tMRCA calculators (McDonald, Walker and McGee) exist based on the infinite alleles model (IAM), but were not yet validated. In this study, the calculators were investigated using our genetic-genealogy database containing 1,126 genealogical pairs with known tMRCA, in which 42 Y-STR markers were analyzed. We observed that the McGee calculator is very discordant with our dataset, while the others give quite similar results. Overall, the tMRCAs from our genealogical pairs are located within the $95 \% \mathrm{Cl}$, but are slightly underestimated for pairs with a close $\mathrm{Y}$-haplotype match, and overestimated when more Y-STR differences are present. Additionally, their large $95 \% \mathrm{Cl}$ are not appropriate for genealogy reconstruction for forensic purposes. To further improve tMRCA estimations, individual Y-STR mutation rates and allele calls should be included to take hidden Y-STR differences into account.
\end{abstract}

Keywords: Ancestor time estimation; MRCA; Y-chromosome; Y-STR; Familial searching 


\section{Introduction}

Familial searching is an investigative identification method in forensic case work where instead of finding the donor of a trace, a relative of the donor may be identified in a pool of men using autosomal or Y-chromosomal DNA analysis. As autosomal DNA kinships fade away over generations due to recombination events, Y-chromosome analysis provides the opportunity to identify patrilineages as $95 \%$ of the chromosome does not undergo recombination. A close or distant biological relative can be verified using (rapidly mutating) short tandem repeats or (RM) Y-STRs, which identifies familial Y-haplotypes [1]. Through Y-haplotype comparison, the time to their most recent common ancestor (tMRCA) can be calculated. The more exact the number of generations can be determined, the easier it becomes to reconstruct the genealogy. In 2001, Walsh used two models to estimate the tMRCA: the infinite alleles model (IAM) indicating that every mutation creates a new allele, and the stepwise mutation model (SMM) which assumes equal probabilities for forward or backward mutations changing only one repeat (one-step) [2]. Walsh identifies no significant differences between both models when at least 20 Y-STRs are analyzed and individuals are separated by at most 200 generations. To date, three online tMRCA calculators exist for genealogy purposes based on a simple form of probability theory taking mainly the IAM into account: McDonald, Walker and McGee. In this study, these calculators are validated for their use in familial searching using our extensive genetic-genealogy database.

\section{Materials and methods}

\subsection{Database}

Our database includes 2,360 males sampled to investigate extrapair paternity rates, haplogroup specific Y-STR mutation rates and parallel Y-STR evolution [3-5]. Permission for DNA analysis and publication was granted by informed consents and approved by the Ethical Commission of University Hospital Leuven (S54010, S55864, S59085). DNA samples were 
collected, extracted and genotyped for $42 \mathrm{Y}$-STRs as described in Claerhout et al. [4]. Data is available through YHRD (https://yhrd.org), under accession numbers YA003651-53, YA003739-42 and YA004300-01.

\section{2 tMRCA calculators}

Three public domain online calculators based on the standard infinite alleles model (IAM) were validated: McDonald [6], Walker [7] and McGee [8]. McDonald input requires three variables: number of Y-STR differences (non-matching alleles), number of analyzed Y-STRs and the average Y-haplotype mutation rate. The obtained output is a probability distribution and a cumulative probability visualized in a list or graph for the number of generations to the MRCA. Walker is a simplistic version of the McDonald calculator whereby only two variables are needed: number of analyzed Y-STRs and the average mutation rate. The output is a probability matrix from zero to five Y-STR mismatches for 1 to 30 generations with the relationship probability or with the number of generations to the tMRCA. The McGee calculator developed by the McGee family is based on their Y-chromosome database, named Y-Utility [8]. Input required are at least two Y-haplotypes with their alleles listed in order of appearance (up to 102 Y-STRs) on the platform. Different calculator settings can be selected by the user: mutation model, output in years or generations, probability percentage, and even the mutation rate being a constant mutation rate by choice, Family Tree DNA derived mutation rates or McDonald mutation rates derived from the Sorenson database. Next to the standard IAM, a hybrid mutation model (HMM) can be selected which is based on the IAM for two Y-STR markers (DYS464 and YCAII) and on the SMM for the other loci. The obtained genetic distance matrix is colored dependent on their relatedness.

\subsection{Validation}

Our previously obtained mean mutation rate $\left(5.03 \times 10^{-3}\right)$ and $42 \mathrm{Y}$-STR markers were used for the McDonald and Walker validation [4]. For the McGee calculator, only 38 Y-STR allele calls 
could be included as three rapidly mutating markers (DYS518, DYS627 and DYF387S1-ab) were not available for selection. McGee was validated using both Family Tree DNA as McDonald mutation rates. These individual Y-STR mutation rates were also compared to our mutation rates [4]. For all calculators, the number of generations with the highest probability was selected and multiplied by two to identify the number of meioses between two biological relatives. This was further compared to the range and average number of generations per number of Y-STR differences in our database.

\section{Results and discussion}

After kinship analysis through extended Y-chromosome comparison, 1,126 biologically confirmed genealogical pairs were identified with known tMRCA ranging from two to 40 generations, altogether counting for 18,175 meioses. Figure 1 visualizes the comparison between our tMRCA data (black) and the calculators (grey). As both McDonald and Walker are based on the same variables (Y-STR differences and average mutation rate), similar results could be observed. The main disadvantage of Walker is that calculations are limited to five mismatches as they conclude that more mismatches are probably irrelevant for most genealogical purposes. Overall, our tMRCAs are located within their broad 95\% confidence interval $(\mathrm{Cl})$, but are slightly underestimated for genealogical pairs with a close Y-haplotype match, and overestimated when more Y-STR differences are present. TMRCA underestimation is caused by allele call ignorance, which disregards hidden mutation events like multiple, parallel or back mutations. tMRCA overestimation is the consequence of the lack of taking individual Y-STR mutation rates into account, calculating Y-STR differences on rapidly mutating markers with an average Y-STR loci mutability rate.

The McGee calculator was, despite the more advanced input of allele calls and their so-called use of individual mutation rates, very discordant with our dataset even though McDonald 
mutation rates followed the same trend as our mutation rates [4]. The latter however turned out to be irrelevant as we observed that tMRCA calculations were still only based on an average mutation rate, since genealogical pairs with equal number of Y-STR differences but on more rapidly mutating markers resulted in the same number of generations. Our tMRCAs did not entirely overlap with their $95 \% \mathrm{Cl}$ and were extremely overestimated for distant genealogical pairs. This again shows the necessity of including individual Y-STR mutation rates and allele calls for more precise tMRCA estimations. Additionally, an adapted mutation model is needed, as IAM ignores hidden multiple, back and parallel mutations and SMM does not take multi-step mutations into account.
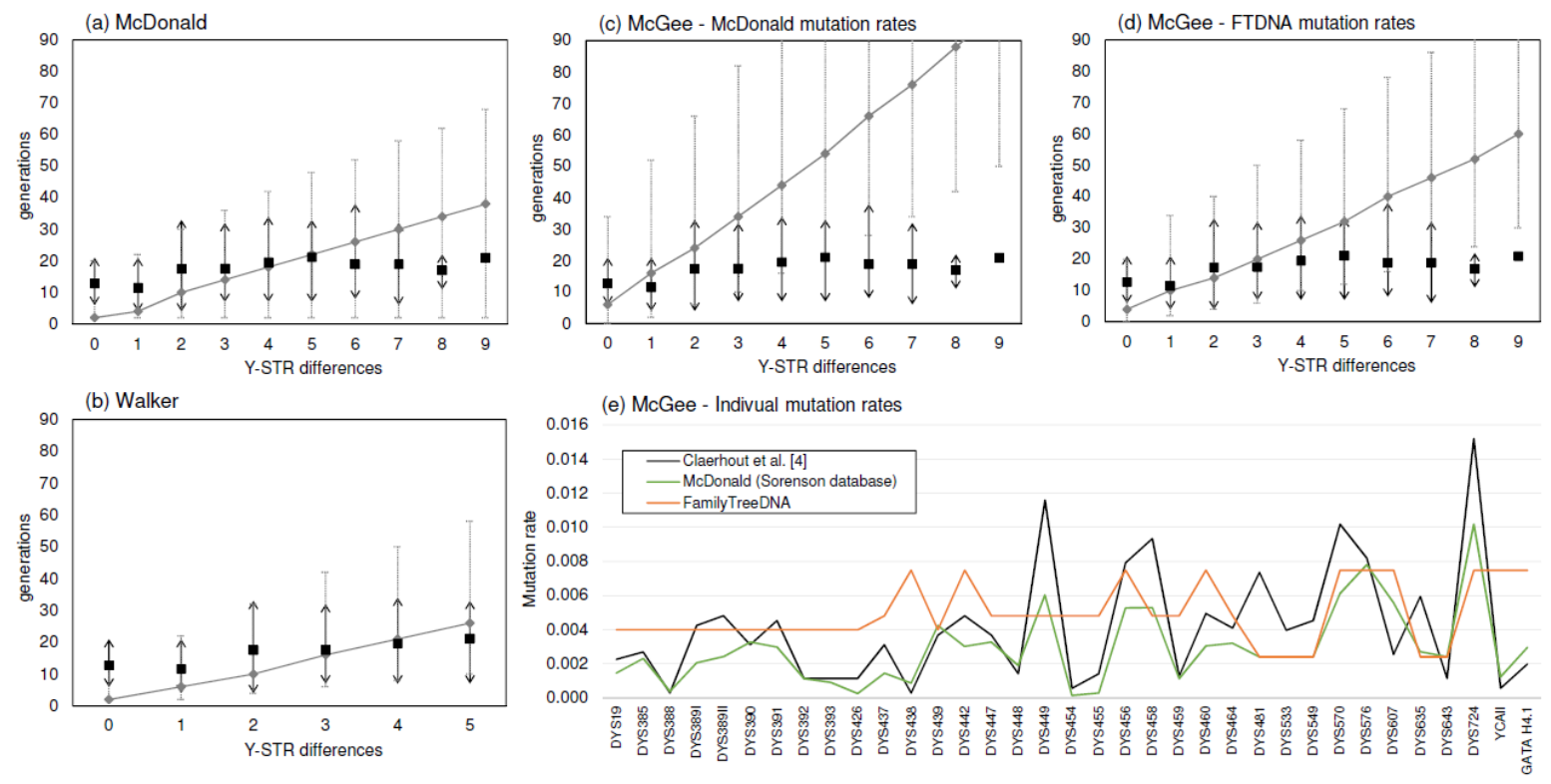

Figure 1: tMRCA calculator validation. (a) McDonald and (b) Walker are based on the same variables. (c-d) McGee uses individual mutation rates adopted from McDonald and Family Tree DNA. (e) Mutation rates comparison [4]. Black: database; grey: calculator.

\section{Conclusion}

Here we demonstrate that the online calculators are not yet appropriate for use in forensic familial searching due to (1) their large $95 \% \mathrm{Cl}$, making predictions too difficult for pedigree reconstructions, and (2) the consistent tMRCA under- and overestimations, leading to 
implausible and dubious probability scores. To further improve tMRCA estimations, a userfriendly calculator should be developed including individual Y-STR mutation rates and allele calls to encounter possible mutational characteristics which could cause divergent results.

\section{Acknowledgements}

We thank all DNA donors for their participation in our genetic-genealogy project. Funding was provided by KU Leuven (BOF-C1 grant C12/15/013).

\section{Conflict of interest statement}

None.

\section{References}

[1] M. Kayser, Forensic use of Y-chromosome DNA: a general overview, Hum. Genet. 136 (2017) 621-635. doi:10.1007/s00439-017-1776-9.

[2] B. Walsh, Estimating the time to the most recent common ancestor for the $Y$ chromosome or mitochondrial DNA for a pair of individuals, Genetics. 158 (2001) 897-912.

[3] M.H.D. Larmuseau, S. Claerhout, L. Gruyters, et al., Genetic-genealogy approach reveals low rate of extrapair paternity in historical Dutch populations, Am. J. Hum. Biol. 29 (2017) 1-9. doi:10.1002/ajhb.23046.

[4] S. Claerhout, M. Vandenbosch, K. Nivelle, et al., Determining Y-STR mutation rates in deep-routing genealogies: Identification of haplogroup differences, Forensic Sci. Int. Genet. 34 (2018) 1-10. doi:10.1016/j.fsigen.2018.01.005.

[5] S. Claerhout, M. Van Der Haegen, L. Vangeel, et al., A game of hide and seq : Identification of parallel Y-STR evolution in deep-rooting pedigrees, Eur. J. Hum. Genet. 27 (2018) 637-646. doi:10.1038/s41431-018-0312-2.

\section{Web references}

[6] J.D. McDonald, online tMRCA Calculator, (2014). http://faculty.scs.illinois.edu/ mcdonald/tmrca.htm.

[7] M. Walker, online tMRCA calculator, (n.d.). http://www.moseswalker.com/mrca/calculator.asp?q=1

[8] D. McGee, Y-Utility: Y-DNA Comparison Utility, FTDNA Mode 111 Marker, (2008). http://www.mymcgee.com/tools/yutility111.html. 